\title{
Acupuncture therapy for chronic low back pain: protocol of a prospective, multi-center, registry study
}

\author{
Xuqiang Wei ${ }^{1}$, Baoyan $\mathrm{Liu}^{2}$, Liyun $\mathrm{He}^{2}$, Xingyue Yang ${ }^{3}$, Jincao Zhou ${ }^{4}$, Hong Zhao ${ }^{4^{*}+}$ and Jia $\mathrm{Liu}^{2^{*+}}$
}

\begin{abstract}
Objective: Acupuncture therapy for chronic low back pain (CLBP) has received increasing attention. Nevertheless, the evidence of efficacy and safety of random controlled trials (RCTs) remains controversial. Acupuncture as a complex intervention influenced by many factors, its effectiveness in treating chronic low back pain in the real world is unknown. We will develop a network-based registry study to evaluate the effectiveness and safety of acupuncture for the treatment of chronic low back pain and explore key factors affecting efficacy in the real world.
\end{abstract}

Methods: A prospective, multi-center and dynamic registry study. All acupuncture related information will be collected through a high-quality structured network platform. Patients with CLBP included in the study met the following criteria: age from 16 to 80 years, using acupuncture as a main therapy and voluntarily signing the informed consent. At least 2000 patients, 27 acupuncturist, and 9 medical centers will be recruited under actual clinical settings at the first stage. Numeric rating scale (NRS), Oswestry Disability Index (ODI) and Effective rate will be measured in pain and functional disability assessment, respectively, as the primary outcome. Evaluation index will be collected at the baseline and follow-up in 1, 4, 12 weeks after the last visit. Hierarchical models and regression analysis will be used to explore the key factors affecting acupuncture effectiveness. Effects between propensity matching groups (Traditional Chinese acupuncture style vs Microacupuncture style, Local acupoint selection vs Non-local acupoint selection, Single Acupuncture vs Combined therapy) will be compared.

Discussion: This study will be conducted based on the characteristics of acupuncture therapy in the "Real World". Fundamental factors affecting the clinical effectiveness of acupuncture and the preferred acupuncture regimen in the treatment of CLBP will be identified. Reliable acupuncture evidence for the treatment of CLBP through the registry will be a significant supplement to the RCTs.

Trial registration: Chinese Clinical Trial Registry, ChiCTR-OOC-17010751 and Acupuncture-Moxibustion Clinical Trial Registry, AMCTR-OOO-17000045. Registered date on 3 December 2016.

Keywords: Acupuncture, Registry, Chronic low back pain, Real-world, Protocol

\footnotetext{
* Correspondence: hongzhaojournal@163.com; marie_liujia@sina.cn

${ }^{\dagger}$ Hong Zhao and Jia Liu contributed equally to this work.

${ }^{4}$ Institute of Acupuncture and Moxibustion, China Academy of Chinese Medical Sciences, No.16 Dongzhimen inside south St. Dongchen District, Beijing 100700, People's Republic of China

${ }^{2}$ Institute of Basic Research in Clinical Medicine, China Academy of Chinese

Medical Sciences, No.16 Dongzhimen inside south St. Dongchen District,

Beijing 100700, People's Republic of China

Full list of author information is available at the end of the article
}

(c) The Author(s). 2019 Open Access This article is distributed under the terms of the Creative Commons Attribution 4.0 International License (http://creativecommons.org/licenses/by/4.0/), which permits unrestricted use, distribution, and reproduction in any medium, provided you give appropriate credit to the original author(s) and the source, provide a link to the Creative Commons license, and indicate if changes were made. The Creative Commons Public Domain Dedication waiver (http://creativecommons.org/publicdomain/zero/1.0/) applies to the data made available in this article, unless otherwise stated. 


\section{Background}

As a consequence of the acceleration of global aging, sedentary lifestyle increasing and average weight gain, chronic low back pain (CLBP) has become a global condition with high incidence. Although disability and financial burden attributed to chronic low back pain is substantially different between countries, the incremental impact of the worldwide health care system predicted to tremendously in the coming decades [1]. According to 2015 global statistics, low back pain accounts for $7.3 \%$ point prevalence of activity-limiting illnesses, affected 5.4 billion people at all ages [2]. CLBP accounts for a significant portion of the $\$ 100$ billion spent annually on treatment in the United States [3]. In China, back pain kept the top one disorder that causes of years lived with disability(YLDs) from 1990 to 2010 [4]. Therefore, effective and safe treatment is especially crucial in overcoming low back pain and disability related to the chronic condition.

In a variety of treatment options devoted to backrelated disability and chronic pain consequence, noninvasive treatment therapies captured more attention. Changes can be verified in recent guidelines for low back pain [5-7]. Interventional procedure and operation are limited to recommend in guidelines, as well as the pharmacotherapy, in consideration of efficacity, safety and accessibility. Moreover, nonpharmacological intervention was recommended as first-line treatment, which included acupuncture, massage, spinal manipulation, and yoga [6]. As a characteristic non-pharmaceutical option against the opioid crisis, acupuncture captured particularly concerning in pain relief. On account of safety and the actual effect, acupuncture is widely accepted in the management of chronic low back pain $[8,9]$. Low back pain was the top 1 frequently treated indication in US's acupuncture clinic according to a cross-sectional study [10]. However, there is a lack of basic dataset on the use of acupuncture to treat CLBP in China and abroad, including the effective population characteristics, reasonable acupuncture session, preferred acupuncture protocol and acupuncture implementation details, etc.

Durable efficacy of acupuncture was reported to improve function and alleviate pain for CLBP patients at the individual level [11]. Nonetheless, contradictory results are subsistent $[12,13]$, that acupuncture efficacy was no better than comforting acupuncture or sham acupuncture [14-16]. Despite negative outcomes of acupuncture for CLBP is not uncommon to discover $[13,14,17]$, controversy, and doubting exist in methodological, research environment, unreasonable comparison, multiple acupuncture interventions and study population [18-20]. Firstly, as a complex intervention featured with specific and nonspecific factors [21], acupuncture is not suiting RCT research paradigm that was initiated more appropriately for drug and biomedically-oriented interventions [20]. RCTs is usually limited to strictly selected patients, highly controlled conditions and using a sham intervention so that results are poorly generalizable to real clinical practice [22, 23]. Secondly, very little evidence for CLBP in the special populations such as elder or some subtype of CLBP [24]. Obviously, under real clinical settings, older adults have a higher incidence of CLBP and are likely to suffer more complications [24, 25]. Furthermore, not only the acupuncture style or type, implementation details, treatment duration and frequency of acupuncture can affect outcomes, but also the experience of the operator, the individual conditions that lead to CLBP and the combined treatment too [26, 27]. The lacking of acupuncture experience and perception in the social context may reduce the effectiveness of acupuncture. Most of RCTs was conducted under noneastern culture, recruited patients lacking acupuncture experience, De Qi perception or cognition were inevitable [28]. Doubtlessly, new research models need to provide more practical details to bridge the gap between rigorous controlled circumstances and real-world practice.

Therefore, we intend to conduct a registry of acupuncture therapy for CLBP through a network platform rooted in the reality of diverse form and operations of acupuncture. This is a prospective, multi-center, registry study. The current status of the study is in recruiting.

The objectives of the study:

- To develop an international registration platform of CLBP for acupuncture therapy;

- To identify the key factors affecting the clinical effectiveness of acupuncture in treating CLBP from the perspective of acupuncture implementation and physicians;

- To analyze population characteristics and subtypes of CLBP suitable for acupuncture.

\section{Methods \\ Study setting}

The Registry project of Acupuncture was firstly proposed and inspired at 8th World Conference on Acupuncture WFAS SYDNEY 2013 [29]. According to the principles and framework demonstrated by the Agency for Healthcare Research and Quality [30]. China Association of Acupuncture-Moxibustion (CAAM) had established an Acupuncture Patient Registration Alliance and initiated a network database platform-International Registry Platform of Acupuncture-Moxibustion (IRPAM) in 2017. Application of browser/server architecture based on cloud platform mode and Oracle database development to construct the interactive network database platform. 
The present protocol partially referenced the guideline of the SPIRIT-PRO Extension Checklist [31] and Registries for Evaluating Patient Outcomes: A User' guide 3th [30]. The technical roadmap is shown in Fig. 1.

\section{Eligibility criteria}

\section{Requirements for recruiting physicians}

Acupuncture practitioners participating in the study should have the following qualifications:

- Engaged in acupuncture related clinical practice > for 5 years;

- Have a certain amount of outpatient volume of patients with CLBP;

- Prepare individual computer can access to the network;

- Volunteer to participate and sign a memorandum of understanding.

\section{Patient source}

Patients with CLBP were enrolled continuously to reduce selection bias. All recruited patients will be registered dynamically by the acupuncture physicians. The diagnostic criteria, inclusion criteria and exclusion criteria as follows.

\section{Diagnostic criteria}

Pain or muscle tension in the area below the chest and above the buttocks, with or without pain in the lower extremities, and the pain lasts for more than 12 weeks [32].

\section{Inclusion criteria}

- Chronic low back pain or acute onset of chronic low back pain were observed as the main objects to meet the diagnostic criteria for chronic low back pain.

- 16 years $\leq$ Age $\leq 80$ years;

- The main treatment method is acupuncture.

- Voluntarily participate in the patient registration study and sign informed consent.

\section{Exclusion criteria}

- Patients with severe systemic diseases, such as primary diseases of cardiovascular, liver, kidney and hematopoietic system, and severe mental disorders;

- Patients with backache caused by tumor, tuberculosis, cyst and other reasons.

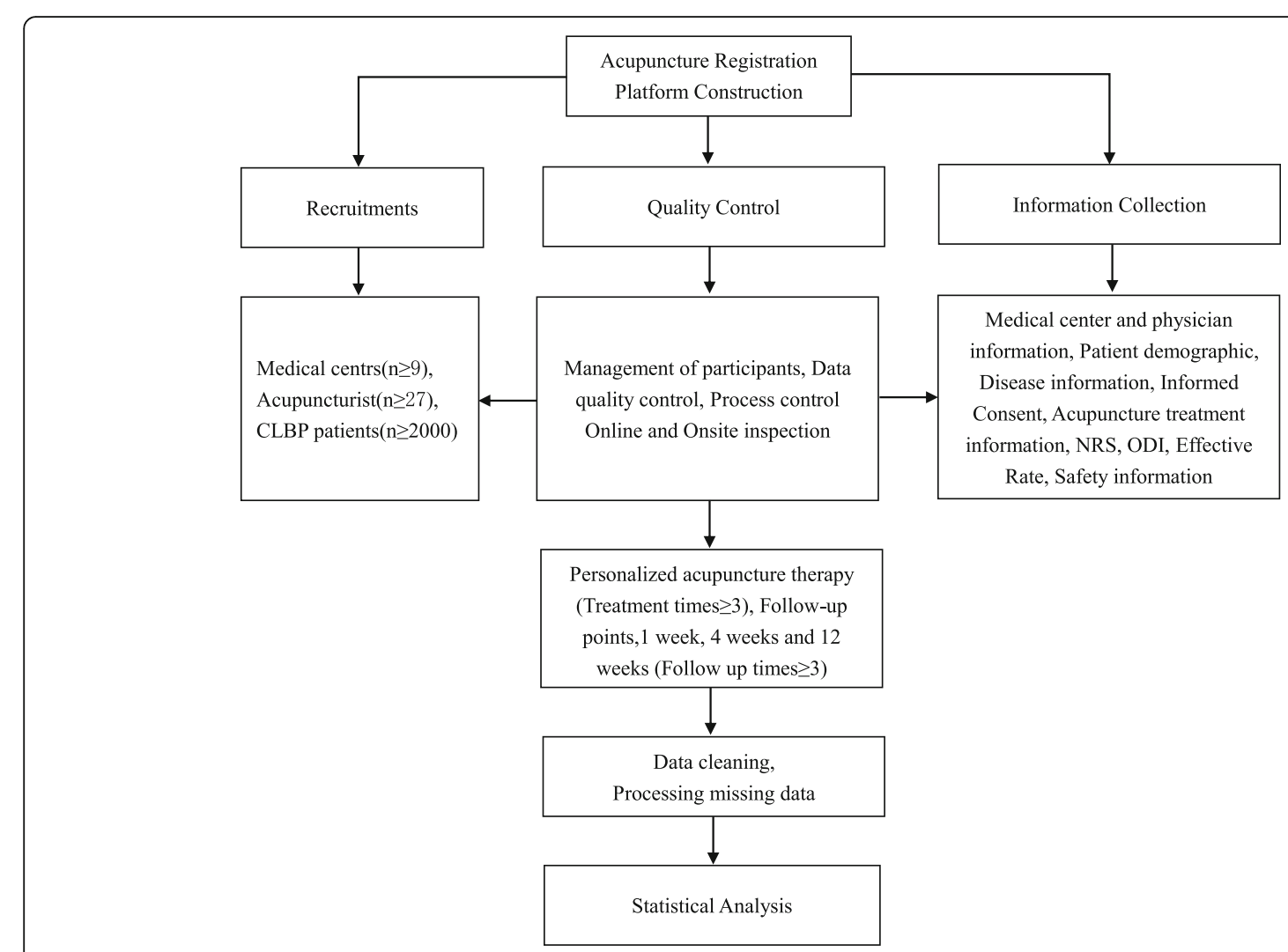

Fig. 1 Flow chart of study 


\section{Outcome measures}

\section{Primary outcomes}

Considering the convenience of real-world measurements, self-reported disability and pain preferable with CLBP. Therefore, Numerical Rating scale (NRS) [33] and Oswestry Disability Index (ODI) [34] were selected as primary outcomes. These two indexes have been confirmed good validity and reliability in the aspects of back-related pain and disability $[35,36]$.

\section{Numerical rating scale (NRS)}

Patients were asked to use the numbers $0-10$ to quantify pain, with ' 0 ' symbolize no pain and ' 10 ' symbolize most pain. Higher the number, heavier the pain.

\section{Oswestry disability index (ODI)}

The ODI instrument contains 1 item on pain and 9 items on the activities of daily living (daily activities, lift weights, walk, sit, stand, sleep, and social activities, travel and sex). Each entry from 0 to 5 points to calculate, the cumulative score will be added 2 times and the total score range of $0-100$ points. Higher the score, lower the back function.

\section{Effective rate}

Minimal clinically important difference (MCID) is an index to identify effective group, that is the NRS score decreased by 2 points or ODI score cut down 10 points [36]. The effective number divides the total number of people included in the analysis is the effective rate.

\section{Secondary indicator Adverse event}

Abnormal conditions in the observation period, such as needle dizziness, broken needles, bleeding and hematoma, will be recorded. The occurrence time, cessation time duration, correlation with acupuncture, the measures taken and outcomes of adverse events will be recorded too. Serious adverse events during the observation will be reported promptly.

\section{Participant timeline}

As a dynamic registry design, we plan to stick to the registry for at least 2 to 5 years. The tolerance range of time is 3 days around the follow-up point. If the patient does not return after the last visit, follow-up is done by phone within 1 week. At least ensure follow-up at these three time points, 1 week, 4 weeks and 12 weeks. Contents of follow-up: whether there is a recurrence, whether there is back pain related surgery, whether there is any other treatment.

\section{Sample size}

Currently, there is no valid sample size calculation method for registry studies. According to experience, the principle of sample size estimation in multivariate analysis of variance is 5-10 times the number of variables. Our preliminary research variables are no more than 20 , so the sample size of the study is $1000-2000$, taking the maximum value, our sample size is set at not less than 2000. To ensure better data representation and reflect the diversity of acupuncture operations, we will recruit no less than 9 centers, at least 3 acupuncturists in each center, that is, at least 27 acupuncturists.

\section{Recruitment}

Through academic conferences of the World Federation of Acupuncture and Moxibustion Societies (WFAS) and Chinese Association of Acupuncture-moxibustion (CAAM), we will attract more physicians. In the recruited centers, recruitment announcements will be launched to help patients know more about the program. Moreover, recruiting more clinicians to register on the platform is an important way that can be conducted on the website (www.amreg.org).

\section{Data elements and collection methods}

All information will be collected through a specific network platform for acupuncture therapy and put on record in a paper version (http://www.amreg.org/). The main data elements include information of medical centers and acupuncturists, characteristics of patients with CLBP, acupuncture operation details, outcomes and safety information. Informed consent requires scanning and uploading. The names of diseases related to chronic back pain are identified by International Classification of Diseases (ICD-10,2010 Version) [37]; TCM syndromes are confirmed by Classification and codes of diseases and ZHENG of traditional Chinese medicine (GB/T 15657-1995) [38]. The acupuncture point vocabulary adopts the WHO Standard Acupuncture Point Locations in the Western Pacific Region (A WPRO Publication, 2008) [39]. All data elements will be collected using the established terminology specifications, data dictionaries.

Most of the data use structured information entry, allowing free text entry to a certain extent. Platform support Pinyin code retrieval and input and fuzzy matching input to ensure the standardization of registration information and facilitate the statistical analysis of data. Under the premise of ensuring the necessary information, entries should be as little as possible to minimize the burden on clinicians. Specific entries and variables are available in Table 1.

Considering the characteristics and clinical practice of acupuncture therapies, we referenced Standards for Reporting Interventions in Clinical Trials of Acupuncture (STRICTA) [27] to building data dictionaries and 
Table 1 Metadata of main items and variables

\begin{tabular}{|c|c|c|c|}
\hline Major data sources & Main items & Property & Main variables and labels \\
\hline \multirow[t]{2}{*}{ Medical Centre } & $\begin{array}{l}\text { Institutional } \\
\text { background }\end{array}$ & Indispensable & Medical Organizations, Location and hospital attributes, Medical institution level \\
\hline & $\begin{array}{l}\text { Registration of } \\
\text { contribution }\end{array}$ & Indispensable & Number of recruited practitioners, Number of enrolled patients \\
\hline \multirow[t]{2}{*}{ Physicians } & $\begin{array}{l}\text { Demographic } \\
\text { Information }\end{array}$ & Indispensable & Name, Age, Gender, Place of residence, Contact information, Educational background \\
\hline & $\begin{array}{l}\text { Practitioner } \\
\text { background }\end{array}$ & Indispensable & $\begin{array}{l}\text { Medical Organizations, Years of employment, Professional qualification (senior or } \\
\text { intermediate professional title) }\end{array}$ \\
\hline \multirow[t]{5}{*}{ Patients } & $\begin{array}{l}\text { Demographic } \\
\text { Information }\end{array}$ & Indispensable & $\begin{array}{l}\text { Name, Age, Gender, Nationality, ID number, Residence, Occupation, Marriage status. } \\
\text { Educational Qualifications, Contact information }\end{array}$ \\
\hline & CLBP Information & Indispensable & $\begin{array}{l}\text { Visit date, Chief complaint, Course of disease, Location of pain, Characteristics of pain, causes } \\
\text { of pain aggravation, Medical history, Causes of pain relief, Back function, Concomitant } \\
\text { symptoms, History of acupuncture and Reception, auxiliary examination }\end{array}$ \\
\hline & $\begin{array}{l}\text { Diagnosis } \\
\text { information }\end{array}$ & Indispensable & $\begin{array}{l}\text { Disease diagnosis, Syndrome differentiation of TCM etiology and meridians, Complicating } \\
\text { disease }\end{array}$ \\
\hline & Lumbar imaging & Optional & X ray, CT examination, Magnetic Resonance Imaging \\
\hline & $\begin{array}{l}\text { Informed } \\
\text { Consent }\end{array}$ & Indispensable & NA \\
\hline \multirow[t]{4}{*}{$\begin{array}{l}\text { Acupuncture } \\
\text { treatment process }\end{array}$} & $\begin{array}{l}\text { Acupuncture } \\
\text { rationale }\end{array}$ & Indispensable & Acupuncture date, Acupuncture style, Acupoint selection principle, Operation of acupoints \\
\hline & $\begin{array}{l}\text { Acupuncture } \\
\text { details }\end{array}$ & Indispensable & $\begin{array}{l}\text { Response elicited, Needle retention time, Needle stimulation, Needle type (gauge, length), } \\
\text { Adjunctive therapy, Needle parameter, Co-intervention }\end{array}$ \\
\hline & $\begin{array}{l}\text { Acupuncture } \\
\text { regimen }\end{array}$ & Indispensable & Number of treatment session, Frequency of treatment \\
\hline & $\begin{array}{l}\text { Combined } \\
\text { therapy }\end{array}$ & Indispensable & Pharmacotherapy, Non-pharmaceutical therapy, Other acupuncture type \\
\hline \multirow[t]{3}{*}{ Outcomes } & $\begin{array}{l}\text { Effectiveness } \\
\text { evaluation }\end{array}$ & Indispensable & NRS, ODI, Effective Rate \\
\hline & Safety evaluation & Indispensable & Adverse events \\
\hline & Follow up & Indispensable & Disease status (Recurrence, Lower back pain related surgery, Seeking other treatments) \\
\hline
\end{tabular}

CT Computed tomography, NA Not applicable, NRS Numeric rating scale, ODI Oswestry disability index, TCM Traditional Chinese Medicine

frameworks. Structured, standardized, and logical metadata will be accumulated to reflect the real clinical process of acupuncture treatment for CLBP. Baseline demographic data, CLBP characteristics, patientcentered outcomes of the effectiveness and safety will be truthfully recorded. Specific data element entry and minimum dataset in the register can be found in Table 2 . Acupuncture treatment and combined treatment should be recorded at each visit. Changes in symptoms, ODI, and NRS scores were recorded before and after treatment for effectiveness evaluation.

\section{Statistical methods Missing data}

Although we have internal quality control methods to ensure data integrity, follow-up nonresponse shall be an inescapable challenge in a prospective observation study. Fortunately, we will recruit experienced physicians with stable patient population (at least 5 years of acupuncture experience or middle and senior titles), so the bias for lack of follow-up could be controlled partially. Real-time monitoring of the electronic network platform shall control missing data less than $20 \%$. According to the classical rules of missing classification, follow-up nonresponse belongs to missing not at random (MNAR) [40]. Multiple imputation invented by Rubin shall be adopted to process missing data [41].

\section{Analytic approach}

Data with poor compliance, non-compliance with the study program or missing important information are not included in the analysis. Data processing and statistical analysis will use SPSS version 24 (IBM Corp., Armonk, NY, USA) and SAS Version 9.4 (SAS Institute Inc., NC 27513-2414, USA).

We will select 1-week,4-week,12-week, assessments respectively at evaluation time point on the ODI and NRS index. Firstly, baseline characteristics comparability needs to be assessed between the propensity-matched groups. Model adjustment suggested by Rosenbaum and Rubin will be adopted to conquer residual covariance imbalances [42]. Secondly, multivariate linear regression 
Table 2 Timetable for data collection

\begin{tabular}{|c|c|c|c|c|c|c|c|c|}
\hline \multirow[t]{3}{*}{ Data elements } & \multicolumn{5}{|c|}{ Therapeutic Session } & \multirow{2}{*}{\multicolumn{3}{|c|}{$\begin{array}{l}\text { Follow-up Period } \\
\text { Minimum follow-up }\end{array}$}} \\
\hline & \multicolumn{3}{|c|}{ Minimum treatment $^{a}$} & \multirow[t]{2}{*}{$\cdots$} & \multirow{2}{*}{$\begin{array}{l}\text { Last } \\
\text { Visit }\end{array}$} & & & \\
\hline & First Visit & Second Visit & Thirdly Visit & & & Week 1 & Week 12 & Week 24 \\
\hline Demographic information & $\checkmark$ & & & & & & & \\
\hline CLBP information & $\checkmark$ & $\checkmark$ & $\checkmark$ & $\checkmark$ & $\checkmark$ & & & \\
\hline Diagnosis & $\checkmark$ & & & & & & & \\
\hline Lumbar imaging & $\checkmark$ & & & & & & & \\
\hline Informed Consent & $\checkmark$ & & & & & & & \\
\hline Acupuncture rationale & $\checkmark$ & & & & & & & \\
\hline Acupuncture details & $\checkmark$ & $\checkmark$ & $\checkmark$ & $\checkmark$ & $\checkmark$ & & & \\
\hline Acupuncture regimen & $\checkmark$ & & & & & & & \\
\hline Combined therapy & $\checkmark$ & $\checkmark$ & $\checkmark$ & $\checkmark$ & $\checkmark$ & & & \\
\hline Numerical Rating scale (NRS) & $\checkmark$ & & & & $\checkmark$ & $\checkmark$ & $\checkmark$ & $\checkmark$ \\
\hline Oswestry Disability Index (ODI) & $\checkmark$ & & & & $\checkmark$ & $\checkmark$ & $\checkmark$ & $\checkmark$ \\
\hline Adverse event & $\checkmark$ & $\checkmark$ & $\checkmark$ & $\checkmark$ & $\checkmark$ & $\checkmark$ & $\checkmark$ & $\checkmark$ \\
\hline Disease status & & & & & & $\checkmark$ & $\checkmark$ & $\checkmark$ \\
\hline
\end{tabular}

${ }^{a}$ The number of acupuncture treatments shall be no less than three times, ${ }^{b}$ No less than three follow-up visits

models will be employed to adjust the propensity score and baseline characteristics. Once matched, change in ODI and NRS between-group at different observation points will be calculated by paired t-test. Thirdly, logistic regression models will be used to evaluate the magnitude of impacts contributed by baseline factors on acupuncture effectiveness for CLBP patients. Descriptive analysis will be used for variables such as the recruited research center,s basic characteristics of doctors and patients, initial diagnosis and intervention details. Quantitative data (Height, Weight, NRS, ODI, etc.) will be described by mean, standard deviation, median, maximum and minimum values. The enumeration data (Acupuncture style, Acupoint selection principle, Operation of acupoints, Effective rate etc.) and ranked data (Educational background, Physician title) will be described by numbers, percentages, rate and constituent ratio. If necessary, parametric methods or alternate nonparametric methods will be selected appropriately.

We will adopt the propensity score matching of patients at 1:1 to imitate an RCT. Baseline confounder will be equilibrated and matched on variables such as demographic data, medical history, duration, intensity, back function. The comparative analysis will be conducted after the baseline unevenness adjustment. Propensity score will match comparison group, such as Traditional Chinese acupuncture style vs Microacupuncture style (Scalp acupuncture, Ear acupuncture, Wrist and ankle acupuncture, Eye acupuncture, Umbilical acupuncture, Abdomen acupuncture), Local acupoint selection vs Non-local acupoint selection and Single Acupuncture vs Combined therapy etc.
Hierarchical models based on the pain grading, medical center, age group, gender, TCM syndromes and classification of diseases will be taken into account to explore the important factors affecting the effectiveness of acupuncture for CLBP [43-45]. Conditional logistic regression models adjusting by different variables to identify predictors of long-term effectiveness presented by ODI and NRS or recurrence at the12-week follow-up.

\section{Quality management}

\section{Management of participants}

Before acupuncture physicians are confirmed to be enrolled, they need to apply for an individual account on the registration platform. Only after the subject research management unit has reviewed their information and approved can they join the project. All organizational personnel, platform developers and acupuncturists are required to participate in standardized training and must acquaint with an investigator's manual.

\section{Data monitoring}

Considering the differences in the filling of registry information between different doctors, standardizing and integrating of diagnosis is the main concern. Data Monitoring will conduct online or on-site quality inspection on the registered information every month, and timely feedback the problems. The concern includes the accuracy, integrity, authenticity and timeliness of the registry. Once problems confirmed, verification or traceability will be conducted timely. Meanwhile, problems existing in the research process will be posted and timely solved via network or telephone. For registered contents that 
are not clearly expressed, such as contradictory information, important data missing. Once verified, online modification will be carried out and recorded for later reference.

\section{Data quality management}

During the data collection process, the platform can proceed with template management, according to the time verification, integrity, validity, and logical verification. Furthermore, the ID number is the unique identification code of the patient on the platform to avoid duplicate entry. The corresponding prompt interface will appear to reduce the wrong data and missing data to facilitate data quality.

Preliminary manual verification of data will be conducted quarterly and timely feedback to the research team. A training conference on registry implementation and problem summary will be conducted every year to gradually summarize the experience. Therefore, the accuracy, fidelity and timeliness of data quality will be improved gradually. A data quality and safety oversight committee will be established to conduct regular assessments of data quality and safety, as well as of acupuncturists. Implement management of procedures, policies, procedures to ensure the quality of data within the registry and control the following errors.

Entry errors: such errors can be avoided through prior data quality checks (such as value range and validity checks), prompt the data that does not conform to the regulation when the system input and strict data cleaning; Biased errors: mainly by checking the continuity of data collection, checking the registered information with the ID and conducting on-site inspections randomly.

\section{Data cleaning and screening}

Cleaning Principles: keeping the original data permanently to guarantee traceability, keeping the original case information unchanged, data were transformed, split, extracted and merged, etc. Specifically, the standardization of the special acupoints, such as 'Huatuo jiaji' will be standardized as 'Waist jiaji point', 'Ashi' was standardized as 'Ashi point'.

\section{Authority management}

To ensure the security and privacy of study data, different access rights will be set for users in the platform. Permissions access mainly contains the operation level and data level, according to different users demands and role in the program. According to the permit classifications, platform will accomplish the access control of operational level and data level. Administrators can check, lock, initialize passwords, set permissions, templates and other basic functions of doctors.

\section{Data security and patient privacy protection}

Data access shall be strictly controlled, and qualification examination shall be carried out for those who log on to the Registry platform. Only researchers and quality inspectors participating in the program have access to the personal medical records of subjects. Clinical registered doctors are only allowed to access their own patients. Sensitive information of patients such as name, ID number and contact information is processed by encryption technology. In data transmission, SSL (Secure Sockets Layer) is used for encryption, and switched virtual network system is used for client access. Encrypted storage of sensitive information of registered doctors and patients, the data should be backed up regularly every week. The completed case information should be archived and stored locally, limiting access rights. The access of storage server also adopts strict authorization mechanism. Each user occupies a unique account in the system and grants different permissions to different researchers.

\section{Dissemination policy}

The research data will be published in the form of a paper after the summary analysis. Different research centers can use the data collected by their own centers to publish, but must pass the review of the research team.

\section{Discussion}

Some registry studies or programs related to low back pain have been established worldwide, such as Quebec registration [46], BOLD registry [25], PRECISION registry [47], etc. Nevertheless, no registration specifically for acupuncture therapy details of CLBP. Unlike previous studies were designed to explain the efficacy of acupuncture. We plan to explore the characteristics of acupuncture implementation and conditions related to more acupuncture benefit for CLBP. Depending on standardized, structured and high-fidelity data collection, we will have a more comprehensive understanding of acupuncture treatment. Through comparisons of different acupuncture stimulation parameters, we will explore the best acupuncture treatment programs for different states and subtypes of CLBP.

Considering maneuverability and convenience of registration implementation, we have carefully considered research design. Logic verification of structured data acquisition and variable input ensures the correctness and completeness of the data. The relatively short follow-up period and convenient data entry process will reduce the data entry burden of clinical acupuncturists and ensure smooth operation. What's more, the STRICTA rules and TCM syndrome standard entries are adopted to ensure data integrity and standardization. 
NRS and ODI scale can be completed in $5 \mathrm{~min}$, the respondent burden is low [35]. Beyond that, ODI and NRS have been widely used in researches [13, 36, 48, 49] for CLBP because of robust psychometric information [35, 50]. We originally intended to cover patients of all ages in the patient group, but considering the actual situation of acupuncture recruitment and the response-ability of the scale, we excluded patients older than 80 years and younger than 16 years. Although many studies have used psychosocial-related scales like Perceived self-efficacy [51], Fear-avoidance beliefs [52], Depression Anxiety Stress Scale $[53,54]$ as indicators or influencing factors for the evaluation of chronic low back pain [43]. We do not adopt these indicators based on some practical considerations. A study has shown that scales supporting acupuncture practice should be considered more often by the acupuncturists to evaluate acupuncture in CLBP [45]. Tedious items and questionnaires of these scales may increase patient resistance and interfere with our judgment on the effectiveness of treatment. Besides, we believe that the psychological factors themselves are embedded in the communication between the acupuncturist and the patients during the acupuncture process. Therefore, the assessment based on the details of the implementation itself also includes the evaluation of psychological related factors. Most importantly, the psychological state and the pain experience itself are difficult to peel off $[51,55]$. Thence, psychological scales are not the preferred indicator for this research study.

Evidence from registry study will be an important supplement to the RCTs. Through the acupuncture registry study for CLBP, we will at least benefit in the following aspects. Firstly, gaining more practical and reliable evidence for acupoint selections and guideline modifications. Secondly, to explore potential confounding factors of acupuncture implementation and provide ideas for high-quality RCTs in the future. Thirdly, through the accumulation of potential acupuncture adverse reactions and excavation of big data, it will be helpful to scientifically choose an individual, safe, and efficient acupuncture style. Characteristics of acupuncture ineffective cases will be identified, avoiding waste of medical resources or delay the patient's condition. Fourthly, the registration organization will help recruit excellent professional acupuncture teams for the treatment of CLBP, providing a better and standardized acupuncture program for the treatment of CLBP. Therefore, our evidence from a large sample, real-world settings and consistent with the characteristics of acupuncture will be indispensable.

\section{Strengths of study}

As far as we know, there are very few registries for acupuncture, which is our biggest innovation. We can obtain responsibly data that relatively high-quality and characterized by acupuncture through structured, standardized, and electronic data collection. Furthermore, prospective data come from real-world will be more convincing than retrospective data. Our registry projects will provide an applicable paradigm for research of complementary and alternative medicine.

\section{Limitations of study}

In the initial stage, center selection bias maybe be inevitably affected by real recruitment results, we will promote geographical representation by expanding recruitment. Lacking the pre-defined comparable group will be the inevitable limitation, as well as selection bias. Although we will adopt the method of propensity score matching to implement post hoc randomization and conduct stratification analysis, hidden, unknown confounding factors will still exist. Perhaps, in future research, we can embed pragmatic randomized controlled trials, pRCTs with long-term follow-up into the platform as a subproject, to obtain more reliable and abundant evidence $[23,56]$.

\section{Conclusion}

This study will identify key factors that influence the effectiveness of acupuncture and explore effective acupuncture interventions with different characteristics of low back pain. Furthermore, reliable acupuncture evidence from the registry will be an important supplement to the future RCTs for the treatment of CLBP.

\section{Abbreviations \\ CAAM: Chinese Association of Acupuncture-moxibustion; CLBP: Chronic low back pain; HIS: Hospital information system; ID: Identification; \\ IRPAM: International Registry Platform of Acupuncture-Moxibustion; MCID: Minimal clinically important difference; MNAR: Missing not at random; NRS: Numeric rating scale; ODI: Oswestry Disability Index; RCTs: Random controlled trials; SSL: Secure Sockets Layer; STRICTA: Standards for Reporting Interventions in Clinical Trials of Acupuncture; TCM: Traditional Chinese Medicine; WFAS: World Federation of Acupuncture-Moxibustion Societies; WPRO: Western Pacific Region Office}

\section{Acknowledgments}

We thank the dissemination and academic support of the Acupuncture Patient Registration Alliance in CAAM. Tanks are also due to the engineers from the Zhong Teng Baimai Baima Medical Technology co.LTD for network technical supporting of the electronic web-based platform.

\section{Authors' contributions \\ $J L$ contributes to the concepts, design and acquired funding for the registry, $\mathrm{HZ}$ take responsibility for clinical administration of the registry and project, and $\mathrm{HZ}$ conceived the study protocol and implementation, XW drafted the original manuscript and contribute to specific daily management of the platform, LH guided the statistical analysis plan, and BL conceived initiative of the registry platform. XY made contributions to the registration of research, and JZ participated in the revision of the protocol. All authors contributed to revising the manuscript and approved the final version to be published.}

Funding

This work was supported by the Tenth independent topic selection in China Academy of Chinese Medical Science (NO. z0470) and National Natural Science Foundation of China (NO. 81704189). The funding body had and will 
not have any role in the design of the study and collection, analysis, and interpretation of the data and writing and submitting manuscripts.

\section{Availability of data and materials}

Data sharing is through the network platform (http://www.amreg.org/), different access rights will be set for users in the platform.

\section{Ethics approval and consent to participate}

The study protocol demonstrated herein was approved by the Ethical inspection committee of the Institute of Basic Research in Traditional Chinese Medicine, Academy of Chinese Medical Sciences. Every recruited patient must provide written informed consent in advance of enrolling

\section{Consent for publication}

Not applicable.

\section{Competing interests}

The authors declare that they have no competing interests.

\section{Author details}

${ }^{1}$ College of Acupuncture and Orthopedic, Hubei University of Chinese Medicine, No.1 Huangjiahu West Road, Hongshan District, Wuhan, Hubei 430065, People's Republic of China. ${ }^{2}$ Institute of Basic Research in Clinical Medicine, China Academy of Chinese Medical Sciences, No.16 Dongzhimen inside south St. Dongchen District, Beijing 100700, People's Republic of China. ${ }^{3}$ School of Acupuncture-Moxibustion and Tuina, Beijing University of Chinese Medicine, No. 11, North Third Ring Road, Chaoyang District, Beijing 100045, People's Republic of China. ${ }^{4}$ Institute of Acupuncture and Moxibustion, China Academy of Chinese Medical Sciences, No.16 Dongzhimen inside south St. Dongchen District, Beijing 100700, People's Republic of China.

Received: 6 September 2019 Accepted: 11 October 2019 Published online: 27 October 2019

\section{References}

1. Hartvigsen J, Hancock MJ, Kongsted A, Louw Q, Ferreira ML, Genevay S, Hoy D, Karppinen J, Pransky G, Sieper J, et al. What low back pain is and why we need to pay attention. Lancet. 2018;391(10137):2356-67.

2. Vos T, Allen C, Arora M, Barber RM, Bhutta ZA, Brown A, Carter A, Casey DC, Charlson FJ, Chen AZ. Global, regional, and national incidence, prevalence, and years lived with disability for 310 diseases and injuries, 1990-2015: a systematic analysis for the global burden of disease study 2015. Lancet. 2016;388(10053):1545-602

3. Dieleman JL, Baral R, Birger M, Bui AL, Bulchis A, Chapin A, Hamavid H, Horst C, Johnson EK, Joseph J. US spending on personal health care and public health, 1996-2013. JAMA. 2016;316(24):2627-46.

4. Yang G, Wang Y, Zeng Y, Gao GF, Liang X, Zhou M, Wan X, Yu S, Jiang Y, Naghavi M, et al. Rapid health transition in China, 1990-2010: findings from the global burden of disease study 2010. Lancet. 2013;381(9882):1987-2015.

5. Bernstein IA, Malik Q, Carville S, Ward S. Low back pain and sciatica: summary of NICE guidance. BMJ. 2017;356:16748.

6. Qaseem A, Wilt TJ, McLean RM, Forciea MA. Noninvasive treatments for acute, subacute, and chronic low back pain: a clinical practice guideline from the American College of Physicians. Ann Intern Med. 2017;166(7): 514-30.

7. Stochkendahl MJ, Kjaer P, Hartvigsen J, Kongsted A, Aaboe J, Andersen M, Andersen M $\varnothing$, Fournier G, Højgaard B, Jensen MB. National clinical guidelines for non-surgical treatment of patients with recent onset low back pain or lumbar radiculopathy. Eur Spine J. 2018;27(1):60-75.

8. Weiss J, Quante S, Xue F, Muche R, Reuss-Borst M. Effectiveness and acceptance of acupuncture in patients with chronic low back pain: results of a prospective, randomized, controlled trial. J Altern Complement Med. 2013;19(12):935-41.

9. Lim TK, Ma Y, Berger F, Litscher G. Acupuncture and neural mechanism in the management of low back pain-an update. Medicines. 2018;5(3). https:// doi.org/10.3390/medicines5030063.

10. Wang H, Yang G, Wang S, Zheng X, Zhang W, Li Y. The most commonly treated acupuncture indications in the United States: a cross-sectional study. Am J Chin Med. 2018;46(07):1387-419.
11. Vickers AJ, Vertosick EA, Lewith G, MacPherson H, Foster NE, Sherman KJ, Irnich D, Witt CM, Linde K, Acupuncture Trialists C. Acupuncture for chronic pain: update of an individual patient data meta-analysis. J Pain. 2018;19(5): 455-74.

12. Wise J. NICE recommends exercise and not acupuncture for low back pain. BMJ. 2016:352:i1765.

13. Thomas KJ, MacPherson H, Thorpe L, Brazier J, Fitter M, Campbell MJ, Roman M, Walters SJ, Nicholl J. Randomised controlled trial of a short course of traditional acupuncture compared with usual care for persistent non-specific low back pain. BMJ. 2006;333(7569):623.

14. Yuan J, Purepong N, Kerr DP, Park J, Bradbury I, Mcdonough S. Effectiveness of acupuncture for low back pain: a systematic review. Spine J. 2008;33(23): E887-900.

15. Haake M, Müller HH, Schadebrittinger C, Basler HD, Schäfer H, Maier C, et al. German acupuncture trials (gerac) for chronic low back pain: randomized, multicenter, blinded, parallel-group trial with 3 groups. Arch Intern Med. 2007:167(17):1892-8.

16. Leibing E, Leonhardt U, Köster G, Goerlitz A, Rosenfeldt JA, Hilgers R, Ramadori G. Acupuncture treatment of chronic low-back pain - a randomized, blinded, placebo-controlled trial with 9-month follow-up. Pain. 2002:96(1-2):189-96.

17. Rubinstein SM, van Middelkoop M, Kuijpers T, Ostelo R, Verhagen AP, de Boer MR, Koes BW, van Tulder MW. A systematic review on the effectiveness of complementary and alternative medicine for chronic non-specific lowback pain. Eur Spine J. 2010;19(8):1213-28.

18. Chia KL. Electroacupuncture treatment of acute low back pain: unlikely to be a placebo response. Acupunct Med. 2014;32(4):354-5.

19. Kim TH, Kang JW, Lee MS. What is lost in the acupuncture trial when using a sham intervention? Acupunct Med. 2017;35(5):384-6.

20. Greco CM, Glick RM, Morone NE, Schneider MJ. Addressing the "it is just placebo" pitfall in CAM: methodology of a project to develop patientreported measures of nonspecific factors in healing. Evid Based Complement Alternat Med. 2013;2013:613797

21. Vas J, Perea-Milla E, Mendez C, Silva LC, Herrera Galante A, Aranda Regules JM, Martinez Barquin DM, Aguilar I, Faus V. Efficacy and safety of acupuncture for the treatment of non-specific acute low back pain: a randomised controlled multicentre trial protocol [ISRCTN65814467]. BMC Complement Altern Med. 2006;6:14.

22. Langevin HM, Hammerschlag R, Lao L, Napadow V, Schnyer RN, Sherman $\mathrm{KJ}$. Controversies in acupuncture research: selection of controls and outcome measures in acupuncture clinical trials. J Altern Complement Med. 2006;12(10):943-53.

23. Luce BR, Kramer JM, Goodman SN, Connor JT, Tunis S, Whicher D, Schwartz JS. Rethinking randomized clinical trials for comparative effectiveness research: the need for transformational change. Ann Intern Med. 2009; 151(3):206-9.

24. Meng CF, Wang D, Ngeow J, Lao L, Peterson M, Paget S. Acupuncture for chronic low back pain in older patients: a randomized, controlled trial. Rheumatology. 2003:42(12):1508-17.

25. Jarvik JG, Comstock BA, Bresnahan BW, Nedeljkovic SS, Nerenz DR, Bauer Z, Avins AL, James K, Turner JA, Heagerty $P$, et al. Study protocol: the Back pain outcomes using longitudinal data (BOLD) registry. BMC Musculoskelet Disord. 2012:13:64.

26. Pach D, Yang-Strobel X, Ludtke R, Roll S, Icke K, Brinkhaus B, Witt CM. Standardized versus individualized Acupuncture for chronic low Back pain: a randomized controlled trial. Evid Based Complement Alternat Med. 2013; 2013:125937.

27. MacPherson H, White A, Cummings M, Jobst K, Rose K, Niemtzow R. Standards for reporting interventions in controlled trials of acupuncture: the STRICTA recommendations. Complement Ther Med. 2001;9(4):246-9.

28. Kong J, Gollub R, Huang T, Polich G, Napadow V, Hui K, Vangel M, Rosen B, Kaptchuk TJ. Acupuncture de qi, from qualitative history to quantitative measurement. J Altern Complement Med. 2007;13(10):1059-70.

29. Liu B. International registration system on clinical cases of acupuncture and moxibustion:thingking and method. In: Book of abstracts of 8th world conference on acupuncture. Sydney: WFAS; 2013. p. 26.

30. Gliklich RE, Dreyer NA, Leavy MB, editors. Registries for evaluating patient outcomes: a user's guide. 3rd ed. Two volumes. (Prepared by the Outcome DEcIDE Center [Outcome Sciences, Inc., a Quintiles company] under Contract No. 290200500351 TO7.) AHRQ Publication No. 13(14)-EHC111. Rockville: Agency for Healthcare Research and Quality; 2014. 
31. Calvert M, Kyte D, Mercieca-Bebber R, Slade A, Chan AW, King MT, Hunn A, Bottomley A, Regnault A, Chan AW, et al. Guidelines for inclusion of patientreported outcomes in clinical trial protocols: the SPIRIT-PRO extension. JAMA. 2018:319(5):483-94.

32. National Guideline C. National Institute for Health And Care Excellence: clinical guidelines. In: Low back pain and sciatica in over 16s: assessment and management, vol. 2016. London: National Institute for Health and Care Excellence (UK) Copyright (c) NICE; 2016.

33. Hartrick $C T$, Kovan JP, Shapiro $\mathrm{S}$. The numeric rating scale for clinical pain measurement: a ratio measure? Pain Pract. 2003;3(4):310-6.

34. Fairbank JCT, Pynsent PB. The Oswestry disability index. Spine J. 2000;25(22): 2940-53.

35. Smeets R, Koke A, Lin CW, Ferreira M, Demoulin C. Measures of function in low back pain/disorders: low back pain rating scale (LBPRS), Oswestry disability index (ODI), progressive Isoinertial lifting evaluation (PILE), Quebec back pain disability scale (QBPDS), and Roland-Morris disability questionnaire (RDQ). Arthritis Care Res. 2011;63(Suppl 11):S158-73.

36. Maughan EF, Lewis JS. Outcome measures in chronic low back pain. Eur Spine J. 2010;19(9):1484-94.

37. International Classification of Diseases (ICD-10) [https://icd.who.int/browse1 0/2010/en]. Accessed 18 Apr 2019.

38. Standardization Administration of the People's Republic of China S, National Administration of Traditional Chinese Medicine. National standards of the People's Republic of China: classification and codes of diseases and ZHENG of traditional Chinese medicine. Beijing: Standards Press of china; 1995.

39. Organization $\mathrm{WH}$. WHO standard acupuncture point locations in the Western Pacific region (a WPRO publication). 1st ed. Manila: World Health Organization; 2008.

40. McPherson S, Barbosa-Leiker C, Mamey MR, McDonell M, Enders CK, Roll J. A 'missing not at random' (MNAR) and 'missing at random' (MAR) growth model comparison with a buprenorphine/naloxone clinical trial. Addiction (Abingdon, England). 2015;110(1):51-8.

41. Rubin DB. Multiple imputation after 18+ years. J Am Stat Assoc. 1996; 91(434):473-89.

42. Rosenbaum PR, Rubin DB. Constructing a control group using multivariate matched sampling methods that incorporate the propensity score. Am Stat. 1985;39(1):33-8.

43. Weidenhammer W, Linde K, Streng A, Hoppe A, Melchart D. Acupuncture for chronic low back pain in routine care: a multicenter observational study. Clin J Pain. 2007:23(2):128-35.

44. Glazov $\mathrm{G}$. The influence of baseline characteristics on response to a laser acupuncture intervention: an exploratory analysis. Acupunct Med. 2010; 28(1):6-11.

45. Stomski NJ, Mackintosh S, Stanley M. Acupuncturists' perspectives on outcome measures to evaluate acupuncture care for chronic low back pain. Complement Ther Med. 2010;18(1):28-41.

46. Choiniere M, Ware MA, Page MG, Lacasse A, Lanctot H, Beaudet N, Boulanger A, Bourgault P, Cloutier C, Coupal L, et al. Development and implementation of a registry of patients attending multidisciplinary pain treatment clinics: the Quebec pain registry. Pain Res Manag. 2017;2017: 8123812.

47. Licciardone JC, Gatchel RJ, Phillips N, Aryal S. The pain registry for epidemiological, clinical, and interventional studies and innovation (PRECISION): registry overview and protocol for a propensity score-matched study of opioid prescribing in patients with low back pain. J Pain Res. 2018; 11:1751-60.

48. Zhang $X$, Wang $Y$, Wang Z, Wang C, Ding W, Liu Z. A randomized clinical trial comparing the effectiveness of electroacupuncture versus mediumfrequency electrotherapy for discogenic sciatica. Evid Based Complement Altern Med. 2017;2017:9502718.

49. Hoy D, March L, Brooks P, Blyth F, Woolf A, Bain C, Williams G, Smith E, Vos T, Barendregt J, et al. The global burden of low back pain: estimates from the global burden of disease 2010 study. Ann Rheum Dis. 2014;73(6):968-74.

50. Deyo RA, Dworkin SF, Amtmann D, Andersson G, Borenstein D, Carragee E, Carrino J, Chou R, Cook K, Delitto A, et al. Report of the NIH task force on research standards for chronic low back pain. Phys Ther. 2015;95(2):e1-e18.

51. Bishop FL, Yardley L, Prescott P, Cooper C, Little P, Lewith GT. Psychological covariates of longitudinal changes in back-related disability in patients undergoing acupuncture. Clin J Pain. 2015;31(3):254-64.

52. McDonough SM, Liddle SD, Hunter R, Walsh DM, Glasgow P, Gormley G, Hurley D, Delitto A, Park J, Bradbury I, et al. Exercise and manual auricular acupuncture: a pilot assessor-blind randomised controlled trial. (The acupuncture and personalised exercise programme (APEP) trial). BMC Musculoskelet Disord. 2008;9:31.

53. Glazov G, Schattner P, Lopez D, Shandley K. Laser acupuncture for chronic non-specific low back pain: a controlled clinical trial. Acupunct Med. 2009; 27(3):94-100.

54. Comachio J, Oliveira Magalhaes M, Nogueira Burke T, Vidal Ramos LA, Peixoto Leao Almeida G, Silva AP, Ferreira de Meneses SR, Costa-Frutuoso JR, Santos Miotto Amorim C, Pasqual Marques A. Efficacy of acupuncture and electroacupuncture in patients with nonspecific low back pain: study protocol for a randomized controlled trial. Trials. 2015;16:469.

55. Ivo R, Nicklas A, Dargel J, Sobottke R, Delank KS, Eysel P, Weber B. Brain structural and psychometric alterations in chronic low back pain. Eur Spine J. 2013;22(9):1958-64.

56. Mullins CD, Whicher D, Reese ES, Tunis S. Generating evidence for comparative effectiveness research using more pragmatic randomized controlled trials. Pharmacoeconomics. 2010;28(10):969-76.

\section{Publisher's Note}

Springer Nature remains neutral with regard to jurisdictional claims in published maps and institutional affiliations.
Ready to submit your research? Choose BMC and benefit from:

- fast, convenient online submission

- thorough peer review by experienced researchers in your field

- rapid publication on acceptance

- support for research data, including large and complex data types

- gold Open Access which fosters wider collaboration and increased citations

- maximum visibility for your research: over $100 \mathrm{M}$ website views per year

At $\mathrm{BMC}$, research is always in progress.

Learn more biomedcentral.com/submissions 